\title{
Binding Enhancment of Antigen-Functionalized PEGylated Gold Nanoparticles onto Antibody-Immobilized Surface by Increasing the Functionalized Antigen using $\alpha$-sulfanyl- $\omega$-amino-PEG
}

\author{
Keitaro Yoshimoto, ${ }^{a, b, c}$ Yuki Hoshino, ${ }^{a}$ Takehiko Ishii, ${ }^{d \neq}$ and Yukio Nagasaki $* a, b, c, e, f$ \\ ${ }^{a}$ Graduate School of Pure and Applied Sciences, University of Tsukuba, Tennoudai 1-1-1, Tsukuba, 305-8573, Ibaraki, Japan. \\ ${ }^{b}$ Center for Tsukuba Advanced Research Alliance (TARA), University of Tsukuba, Tennoudai 1-1-1, Tsukuba, 305-8573, Ibaraki, Japan. \\ ${ }^{c}$ Tsukuba Research Center for Interdisciplinary Materials Science (TIMS),University of Tsukuba, Tennoudai 1-1-1, Tsukuba, 305-8573, \\ Ibaraki, Japan. \\ ${ }^{d}$ Department of Materials Science and Technology, Tokyo University of Science, Yamazaki 2641, Noda 278-8510, Chiba, Japan. \\ ${ }^{e}$ Masters School of Medical Science, Graduate School of Comprehensive Human Sciences, University of Tsukuba, Tennoudai 1-1-1, \\ Tsukuba, 305-8573, Ibaraki, Japan. \\ ${ }^{f}$ Satellite Laboratory, International Center for Materials Nanoarchitectonics (MANA), National Institute of Materials Science (NIMS), \\ Sengen 1-2-1, Tsukuba, 305-0047, Ibaraki, Japan. \\ Fax: 81-029-853-5749; Tel: 81-029-853-5749; E-mail: nagasaki@nagalabo.jp \\ $\ddagger$ Present address: Department of Bioengineering, School of Engineering, University of Tokyo, 7-3-1 Hongo, Bunkyo-ku, 113-8656, \\ Tokyo, Japan.
}

\section{Experimental section}

Materials. Commercial tetrahydrofuran (THF; Kanto Chemical, Tokyo, Japan) and allyl alcohol (Wako Pure Chemical Industries, Ltd., Osaka, Japan) were purified by conventional methods. ${ }^{1}$ Ethylene oxide (EO; Sumitomo-Seika Chemicals Co. Ltd., Osaka, Japan) was dried over calcium hydride (Wako Pure Chemical Industries, Ltd., Osaka, Japan) and distilled under an argon atmosphere. Potassium naphthalene was used as a THF solution, whose concentration was determined by titration. Succinic anhydride (Wako Pure Chemical Industries, Ltd., Osaka, Japan) was recrystallized from THF and washed with diethyl ether (Kanto Chemical, Tokyo, Japan). After drying it in vacuo and then dissolved it in dry THF $(0.7 \mathrm{M})$. Azoisobutyronitrile (AIBN; Wako Pure Chemical Industries, Ltd., Osaka, Japan) was recrystallized from methanol (Kanto Chemical, Tokyo, Japan) and dried in vacuo. Thioacetic acid, 2,2'-dithiodipyridine (2-PDS; Tokyo Kasei Kogyo Co., Ltd., Tokyo, Japan), n-propylamine (Wako Pure Chemical Industries, Ltd., Osaka, Japan), fluorescein isothiocyanate (FITC; Dojindo Laboratories, kumamoto, Japan) and all other reagents were used as received. Thiol-ended PEG derivatives (Acetal-PEG-SH) were synthesized according to our previous works. ${ }^{2}$ A gold chip (SIA KIT Au) for SPR measurement was purchased from GE Healthcare Bio-Sciences KK (Tokyo, Japan). Bovine serum albumin (BSA) and fluorescein-labeled BSA (FITC-BSA) were purchased from Sigma-Aldrich Fine Chemicals (St Louis, Missouri, USA). Anti-fluorescein antibody (Goat-Poly, $\left.\mathrm{F}\left(\mathrm{ab}{ }^{\prime}\right)_{2}\right)$ was obtained from Rockland Immunochemicals, Inc (Gilbertsville, Pennsylvania, USA). Gold nano particle $(10 \mathrm{~nm})$ was purchased from BB International Ltd. (Cardiff, UK). SPR evaluations were carried out on a Biacore 3000 device (GE Healthcare Bio-Sciences KK, Tokyo, Japan). Transmission electron microscope images of PEGylated gold nanoparticles were obtained by energy filtered transmission electron microscope (EF-TEM) LEO 922 OMEGA (Carl Zeiss, Jena, Germany). The water used in this study was purified using a Milli-Q system (Nihon Millipore Co., Tokyo, Japan).

\section{Synthesis of HS-PEG-NH 2 (6).}

Preparation of Allyl-PEG-NH $\mathrm{NH}_{2}$ (4). $4\left(M_{\mathrm{w}}=5,000\right)$ was synthesized according to the literature ${ }^{3}$. The products were purified by column chromatography on CM sephadex C-50 (weak cation exchange chromatography). After removal of the solvent by rotary evaporation, the fractionated polymer was finally obtained by freeze drying. The weight-average 
molecular weight and the polydispersity of PEG-DMA were 5200 and 1.04, respectively.

Preparation of Thioacetate-PEG- $\mathrm{NH}_{2}$ (5). ${ }^{4}$ Thioacetate-PEG- $\mathrm{NH}_{2}$ was synthesized and obtained as thioacetate-PEG-NH${ }_{3} \mathrm{Cl} .1 .0 \mathrm{~g}(0.2 \mathrm{mmol})$ of 4 was dissolved in $0.1 \mathrm{M}$ hydrochloric acid and freeze dried to remove the solvents. The obtained allyl-PEG- $\mathrm{NH}_{3} \mathrm{Cl}(0.2 \mathrm{mmol}, 1 \mathrm{~g})$ was dissolved in $140 \mathrm{~mL}$ of methanol, together with 20 mmol of thioacetic acid and $0.02 \mathrm{mmol}$ of AIBN under argon atmosphere. The solution was irradiated for $15 \mathrm{~h}$ with ultraviolet rays light $\left(300 \mathrm{~mW} / \mathrm{cm}^{2}\right.$, at $\left.365 \mathrm{~nm}\right)$. Then it was precipitated in ether and freeze-dried to remove the solvents (yield: $98 \%$ ). As shown in Figure S2, ${ }^{1} \mathrm{H}-\mathrm{NMR}$ spectrum of the product indicated that the proton signals of allyl group disappeared completely, while new signals of thioacetate group appeared.

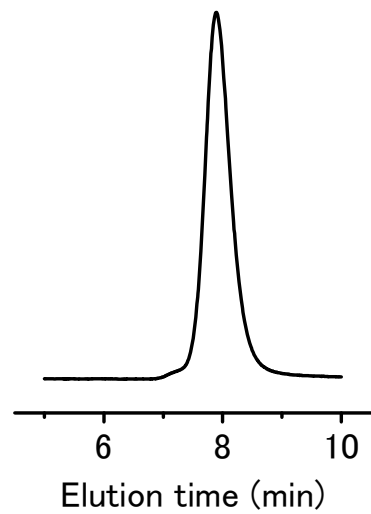

Figure S1 Chromatogram of 5. Size exclusion chromatography measurements were performed on a Tosoh HLC-8120 gel permeation chromatography instrument (Tosoh, Tokyo, Japan) equipped with two consecutive gel columns, TSK-Gel SUPER HZ3000 and TSK-Gel SUPER HZ2500 (Tosoh Co., Tokyo, Japan), and an internal refractive index (RI) detector (TOSOHHLC-8020RI), using THF containing $0.5 \mathrm{wt} \%$ triethylamine as an eluent at a flow rate of $0.35 \mathrm{~mL} / \mathrm{min}$ at $40{ }^{\circ} \mathrm{C}$.

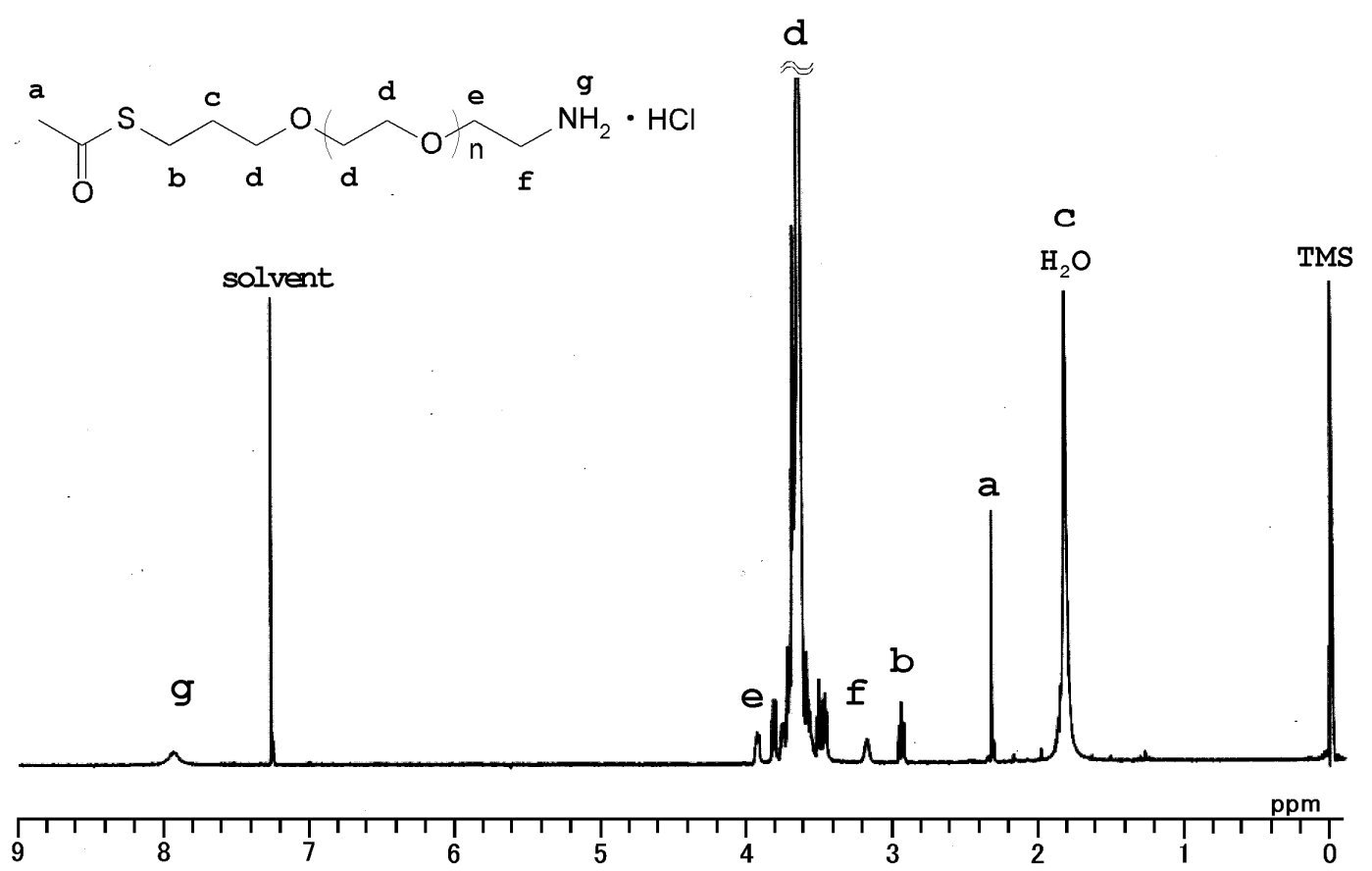

Figure S2 ${ }^{1} \mathrm{H}-\mathrm{NMR}$ spectrum of $\mathbf{5}$ in $\mathrm{CDCl}_{3}$ at r.t. ${ }^{1} \mathrm{H}-\mathrm{NMR}$ spectrum was measured on a JEOL EX-400 instrument (JEOL Ltd., Tokyo, Japan) at $400 \mathrm{MHz}$. 
Preparation of HS-PEG-NH $\mathrm{H}_{2}(6) . \quad 0.5 \mathrm{~g}(0.1 \mathrm{mmol})$ of 5 was dissolved in $5 \mathrm{~mL}$ of sodium methoxide-methanol solution and stirred for $30 \mathrm{~min}$ at room temperature. After removal of the solvent by rotary evaporation, the received product was dissolved in $3 \mathrm{~mL}$ of $1 \mathrm{M}$ hydrochloric acid. The aqueous $\mathrm{HCl}$ phase was extracted three times with chloroform. The organic phase was dried with sodium sulfate followed by filtration. After removal of the solvent by rotary evaporation, the polymer sample collected was subjected to freeze drying with benzene to remove the solvents. HS-PEG- $\mathrm{NH}_{2}$ was thus obtained, and the yield was $98 \%$.

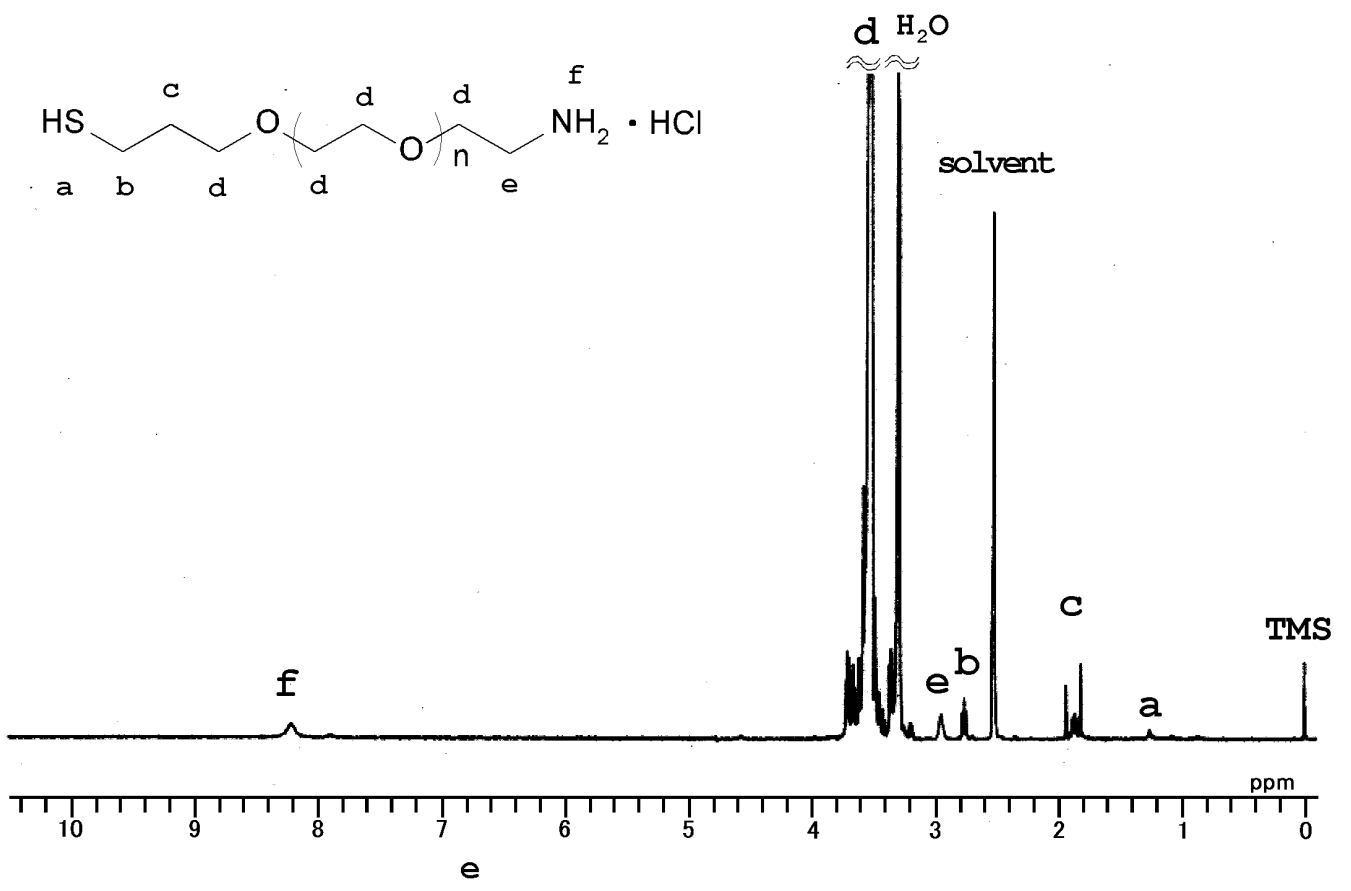

Figure S3 ${ }^{1} \mathrm{H}-\mathrm{NMR}$ spectrum of 6 HS-PEG-NH${ }_{3} \mathrm{Cl}$ in DMSO- $d_{6}$ at $40{ }^{\circ} \mathrm{C}$. Other experimental conditions were the same as descried in Figure S2.

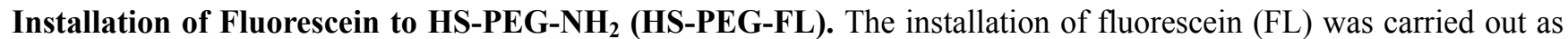

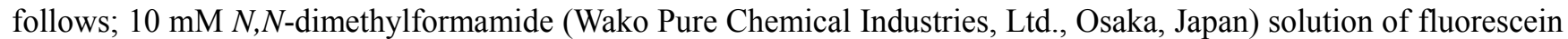
isothiocyanate (10 eq.) was added to the solution of HS-PEG- $\mathrm{NH}_{2}\left(M_{\mathrm{w}}=5,000\right)$ in $100 \mathrm{mM}$ carbonate buffer solution (pH 9.0) and stirred for 1 day at room temperature. The polymer was precipitated in ether and removed the solvents by rotary evaporation. After the reaction, crude mixture solution was purified by a size exclusion column (sephadex G-25) and then removed the solvent by freeze drying. The terminal-functionalization was confirmed by ion exchange high performance liquid chromatography (HPLC) and ${ }^{1} \mathrm{H}-\mathrm{NMR}$ spectrum as shown in Figures S4 and 5, respectively. Almost quantitative functionalization of FITC was confirmed. 


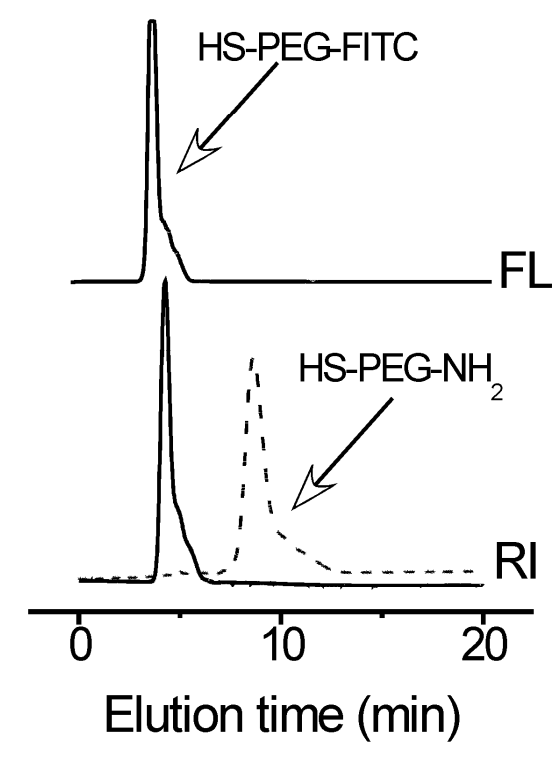

Fig S4 Chromatograms of HS-PEG-NH $\mathrm{NH}_{2}$ (dashed line) and HS-PEG-NH $\mathrm{N}_{2}$ reacted with FITC (solid line, HS-PEG-FL) monitored by fluorescence detection (ex. at $490 \mathrm{~nm}$ and em. at $520 \mathrm{~nm}$, top) and reflective index (bottom). Ion exchange chromatography measurements were performed on a JUSCO LC-2000 plus (Jusco Co., Ltd, Tokyo, Japan) equipped with a TSK-GEL SP-5PW column (Tosoh Co., Tokyo, Japan), using 2 mM phosphate buffer (pH6.5) as an eluent at a flow rate of $0.5 \mathrm{~mL} / \mathrm{min}$ at $40^{\circ} \mathrm{C}$.

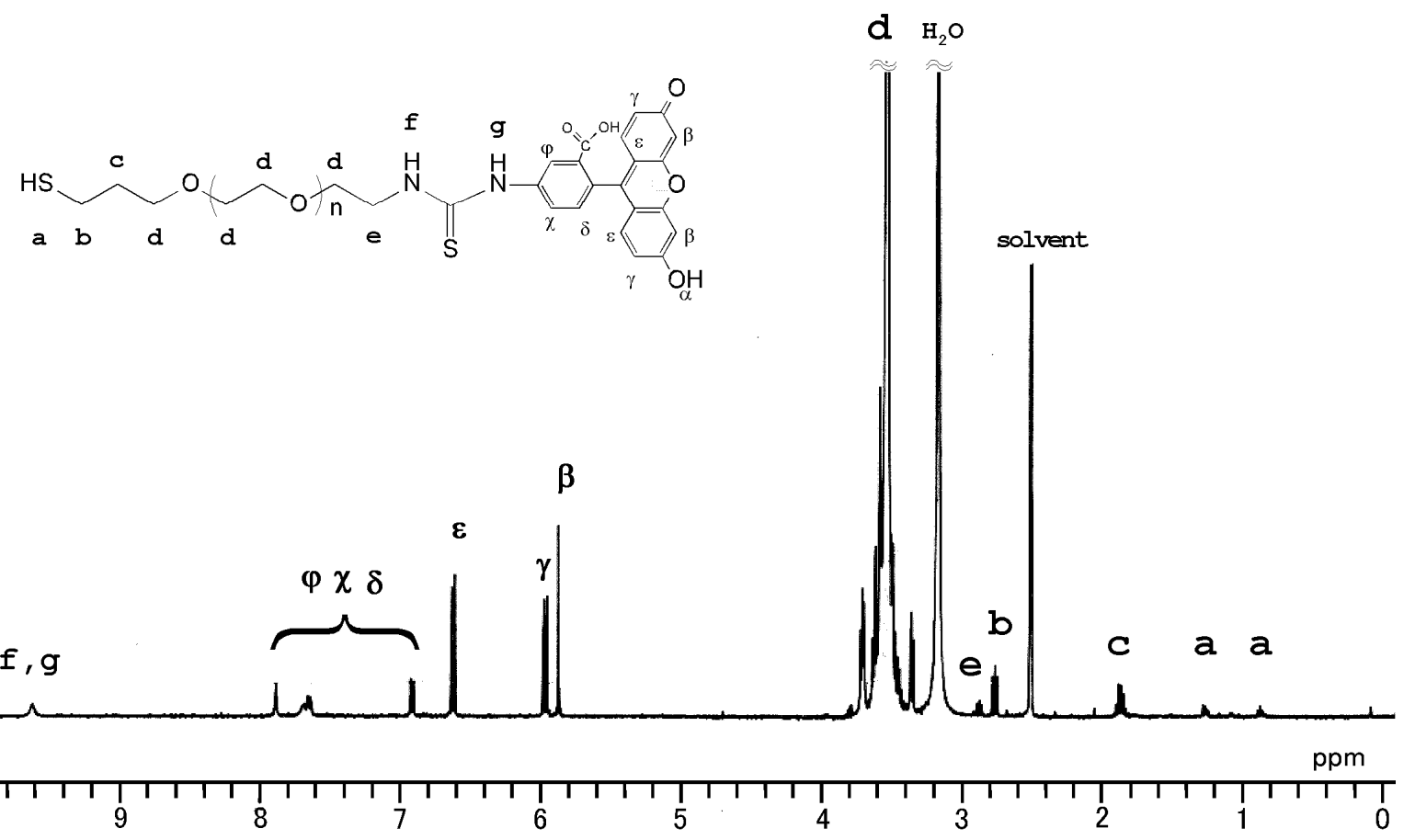

Figure S5. ${ }^{1} \mathrm{H}-\mathrm{NMR}$ spectrum of HS-PEG-FL in DMSO- $d_{6}$ at $60{ }^{\circ} \mathrm{C}$. Experimental conditions were the same as descried in Figure S2. 
Preparation of FL-GNP with various density of FL on PEG surface. Given ratio of HS-PEG-FL (5k) and acetal-PEG-SH (5k) mixture solution as shown in Table1 was added to $10 \mathrm{~mL}$ of $9.47 \mathrm{pM}$ colloidal gold solution under PEG-SH/GNP molar ratio of $7.8 \times 10^{3}$ and stirred for $1 \mathrm{~h}$ at room temperature. The temperature of mixture was increased to $70{ }^{\circ} \mathrm{C}$ and stirred for another $1 \mathrm{~h}$ to increase the PEG brushes density. PEGylated GNPs thus prepared were precipitated by centrifugation $\left(100,000 \times \mathrm{g}, 25{ }^{\circ} \mathrm{C}, 25 \mathrm{~min}\right)$ and then washed with water. This precipitate/redispersion cycle was repeated 5 times to remove unmodified PEG.

Estimation of the immobilized number of FL-PEG-SH on GNP surface by fluorescence based analysis. The immobilized amounts of HS-PEG-FL on the GNP surface were quantified by fluorescence-based analysis as follows ${ }^{5}$; $10 \mathrm{mM}$ sodium cyanate (Kanto Chemical, Tokyo, Japan) solution was added FL-PEG-GNP solution in $100 \mathrm{mM}$ carbonate buffer ( $\mathrm{pH} \mathrm{10}$, final $\mathrm{CN}^{-}$concentration was adjusted to $1 \mathrm{mM}$ ). Then the fluorescence intensities derived from fluorescein at $510 \mathrm{~nm}$ were measured by fluorescence spectroscopy after centrifugation (the absorption band of GNP around $520 \mathrm{~nm}$ was completely disappeared). On the other hand, the concentrations of GNPs were determined by atomic adsorption spectroscopy. The numbers of modified HS-PEG-FL per GNP as shown in Table 1 were determined using a calibration curve of fluorescein in $100 \mathrm{mM}$ carbonate buffer solution.

Transmission Electron Microscope (TEM) image. TEM image for FL-GNP was prepared as follows: HS-PEG-FL (5k) was mixed to colloidal gold at the HS-PEG-FL/GNP molar ratio of $7.8 \times 10^{3}$ and stirred for $1 \mathrm{~h}$ at room temperature. Then the temperature of mixture was increased to $70{ }^{\circ} \mathrm{C}$ and stirred for another $1 \mathrm{~h}$. Subsequently, MeO-PEG-SH (2k) solution was added to the mixture at the MeO-PEG-SH/GNP molar ratio of $7.8 \times 10^{2}$ and stirred for $1 \mathrm{~h}$ at $70^{\circ} \mathrm{C}$. Thus prepared FL-GNP was precipitated by centrifugation $\left(100,000 \times \mathrm{g}, 25^{\circ} \mathrm{C}, 25 \mathrm{~min}\right)$, then washed with purified water. Purification of obtained GNP was carried out using same procedure as described above. The number of modified HS-PEG-FL per GNP surface was quantified by the same procedure as described above and it was determined to be 47. For EF-TEM images, a drop of dilute solution of a FL-PEG-GNP was placed on a formal film-coated $\mathrm{Cu}$ grid and allowing to dry in air. The observation of EF-TEM was carried out at $200 \mathrm{kV}$.

Preparation of Fab'/PEG-SH co-immobilized sensor surface. Fab' fragmentized anti-fluorescein antibody was prepared just prior to use as follows; $12 \mu \mathrm{L}$ of $10 \mathrm{mM}$ dithiothreitol solution was added to $200 \mu \mathrm{l}$ of $9.5 \mu \mathrm{M} \mathrm{F}\left(\mathrm{ab}^{\prime}\right)_{2}$ anti-fluorescein antibody in $100 \mathrm{mM}$ phosphate buffer containing $5 \mathrm{mM}$ ethylene diamine tetraacetic acid (EDTA) $(\mathrm{pH}$ 6.5) and then the mixture was stood for $2 \mathrm{~h}$ at room temperature. The purification of the antibody Fab' fragment was carried out by centrifugation on a Microcon YM-30 column (Nihon Millipore Co., Tokyo, Japan) using $100 \mathrm{mM}$ phosphate buffer containing $5 \mathrm{mM}$ ethylene diamine tetraacetic acid (EDTA) $(\mathrm{pH} 6.5)\left(14,000 \times \mathrm{g}, 4^{\circ} \mathrm{C}, 30 \mathrm{~min}\right)$. The concentration of antibody Fab' fragment was adjusted by measuring the absorbance at $280 \mathrm{~nm}$.

The SPR sensor chips were cleaned up in a UV/ozone cleaner for 15 min just prior to use and the cleaned sensor chips were docked into the SPR instrument. $1 \mu \mathrm{M}$ Fab' anti-fluorescein antibody in $100 \mathrm{mM}$ phosphate buffer solution ( $\mathrm{pH} 7.4$, containing $150 \mathrm{mM} \mathrm{NaCl}$ and $5 \mathrm{mM}$ EDTA) was flowed to the gold surface at a constant flow rate at 5 $\mu \mathrm{L} / \mathrm{min}$ until SPR responses reached to given values. As a blocking agent, $0.1 \mathrm{mg} / \mathrm{mL}$ of PEG-SH (2k) solution was flowed to the Fab' modified gold surface at a constant flow rate of $5 \mu \mathrm{l} / \mathrm{min}$ for $30 \mathrm{~min}$ at $25^{\circ} \mathrm{C}$.

\section{Supporting Data}

We confirm the reproducibility in the nondissociative binding property of the constructed GNPs with high-level antigen functionalities. And the differences in the SPR angle shifts between the PEGyated GNPs with high-level antigen functionalities and that of low-level functionalities are significant as described in Figure 2b and Figure S6. 


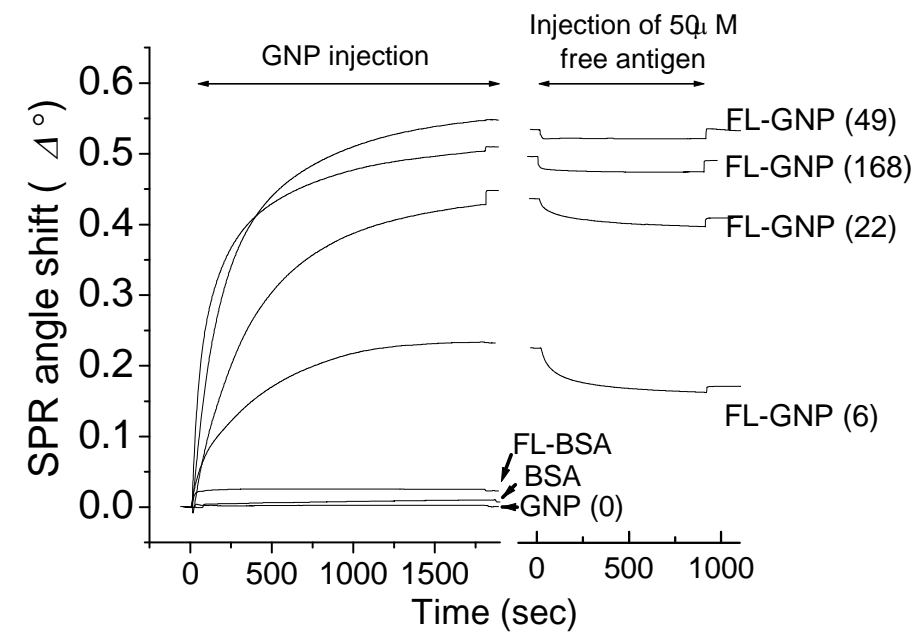

Figure S6 SPR sensorgrams for the association/dissociation behavior of $5 \mathrm{nM}$ FL-GNPs(0)-(168) onto the Fab'/PEG-SH(2k) co-immobilized sensor surface. All samples and running buffer solutions were prepared with 50 $\mathrm{mM}$ sodium phosphate containing $150 \mathrm{mM} \mathrm{NaCl}$ at $\mathrm{pH}$ 7.4. Sample injections were carried out at a constant flow rate of $5 \mu \mathrm{L} / \mathrm{min}$ at $25^{\circ} \mathrm{C}$. Replacement reaction of FL-GNP(n) with FL was carried out by injecting of $50 \mu \mathrm{M}$ FL solution.

\section{References}

1. D. D. Perrin, W. L. F. Armarego, D. R. Perrin, Purification of Laboratory Chemicals. 1980

2. Y. Akiyama, H. Otsuka, Y. Nagasaki, M. Kato, and K. Kataoka, Bioconjugate Chem., 2000, 11, 947-950.

3. T. Matsuya, S. Tashiro, N. Hoshino, N. Shibata, Y. Nagasaki, and K. Kataoka; Anal. Chem., 2003, 75, 6124.-6132.

4. (a) S. Herrwerth., T. Rosendahl, C. Feng, J. Fick, W. Eck、M. Himmelhaus, R. Dahint, and M. Grunze, Langmuir, 2003, 19, 1880-1887; (b) T. Ishii, M. Yamada, T. Hirase, and Y. Nagasaki, Polymer J., 2005, 37, 221-228.

5. L. M. Demers, C. A. Mirkin, R. C. Mucic, R. A. Reynolds, III, R. L. Letsinger, R. Elghanian and G. Viswanadham; Anal. Chem., 2000, 72, 5535-5541. 